Revue d'histoire de l'Amérique française

REVUE D.HISTOIRE DE L'AMÉRIQUE FRANÇAISE

HERON, Craig et Steve PENFOLD, The Workers' Festival. A History of Labour Day in Canada (Toronto, University of

Toronto Press, 2005), 340 p.

\title{
Martin Petitclerc
}

Volume 60, numéro 1-2, été-automne 2006

L'histoire environnementale

URI : https://id.erudit.org/iderudit/014604ar

DOI : https://doi.org/10.7202/014604ar

Aller au sommaire du numéro

Éditeur(s)

Institut d'histoire de l'Amérique française

ISSN

0035-2357 (imprimé)

1492-1383 (numérique)

Découvrir la revue

Citer ce compte rendu

Petitclerc, M. (2006). Compte rendu de [HERON, Craig et Steve PENFOLD, The Workers' Festival. A History of Labour Day in Canada (Toronto, University of Toronto Press, 2005), 340 p.] Revue d'histoire de l'Amérique française, 60(1-2), 218-221. https://doi.org/10.7202/014604ar services d'Érudit (y compris la reproduction) est assujettie à sa politique d'utilisation que vous pouvez consulter en ligne.

https://apropos.erudit.org/fr/usagers/politique-dutilisation/ 
semblé plus « ouverte» que l'histoire à l'origine, mais par la suite, l'entrée des femmes en histoire s'est faite plus facilement qu'en physique.

Jean Mackenzie Leiper examine les expériences des étudiantes en droit de plusieurs facultés ontariennes, sous l'angle de la classe et du genre. Elle observe que ce milieu de formation est lent à se défaire de sa séculaire tradition de "society of learned gentlemen".

Deux chapitres examinent deux professions traditionnellement féminines et abordent des questions plus étroites. Linda J. Quincy analyse l'impact des infirmières volontaires, durant la Première Guerre mondiale, sur le développement de la profession d'infirmière. Tracey L. Adams rapporte l'évolution de la profession d'hygiénistes dentaires en Ontario, laquelle, apparue dans la faculté d'art dentaire, a été mutée au niveau du «vocational college» durant les années 1970. En quête de statut et d'autonomie, les hygiénistes dentaires travaillent en ce moment à transformer leur formation en baccalauréat. Toutefois, on comprend mal pourquoi les directrices ont choisi d'inclure dans leur ouvrage un chapitre sur le «birth control» aux Indes durant les années 1930. Cette problématique, par le caractère hautement controversé à l'époque, est plutôt éloignée de l'ensemble du volume.

En dépit de la variété des perspectives adoptées, cet ouvrage ouvre d'intéressantes perspectives de recherche. On ne peut que regretter que de telles études n'aient pas encore été entreprises au Québec, ailleurs que dans le champ du domaine paramédical, par les études de Nadia FahmyEid. Des études particulières sur la médecine, le droit, le service social, entre autres, sont en ce moment disponibles et une vue d'ensemble sur la situation québécoise permettrait de vérifier si les constats observés au Canada anglais se retrouvent au Québec.

\section{Micheline Dumont \\ Professeure émérite Université de Sherbrooke}

HERON, Craig et Steve PENFOLD, The Workers' Festival. A History of Labour Day in Canada (Toronto, University of Toronto Press, 2005), 340 p.

The Workers' Festival, écrit par Craig Heron - spécialiste de l'histoire ouvrière canadienne - et Steve Penfold - spécialiste de l'histoire de la consommation et de la culture populaire - vise à retracer les grandes mutations culturelles ayant marqué la fête du Travail depuis la fin du 
$\mathrm{xIX}^{\mathrm{e}}$ siècle. L'objectif du livre est d'analyser la fête du Travail, phénomène complexe qui condense une foule d'enjeux politiques, économiques et sociaux, à partir de cette manifestation culturelle qu'est le défilé. Les auteurs soutiennent que l'étude de la "plus importante création culturelle ouvrière de l'histoire canadienne» est essentielle pour comprendre, plus largement, l'histoire culturelle du Canada. L'ouvrage déborde ainsi les thèmes traditionnels de l'histoire ouvrière pour s'ouvrir sur des problématiques liées à l'espace public et à la société de consommation.

Le premier chapitre du livre, qui en contient six, vise à retracer les origines culturelles de la fête du Travail. Heron et Penfold insistent sur les nombreux emprunts faits à l'égard des célébrations publiques victoriennes (Victoria Day, Dominion Day) et des célébrations catholiques comme la fête de la Saint-Jean-Baptiste qui auraient donné la structure culturelle de "respectabilité » à la fête du Travail à partir de la fin du $\mathrm{XIX}^{\mathrm{e}}$ siècle. Dans le deuxième chapitre, les auteurs analysent les premières décennies de la fête du Travail, après sa reconnaissance officielle en 1894 jusqu'à la Première Guerre mondiale. C'est l'âge d'or du spectacle des travailleurs de métiers qui sont regroupés au sein des unions internationales américaines. Ce spectacle ordonné est profondément marqué par le souci de respectabilité et de reconnaissance sociale de ces travailleurs qualifiés qui désirent rappeler leur contribution au progrès industriel. Cette reconnaissance a toutefois un prix car le discours de la respectabilité trace des frontières artificielles à l'intérieur de la classe ouvrière, opposant notamment les travailleurs de métiers aux ouvriers sans qualification, aux femmes et aux immigrants.

Les deux chapitres suivants s'intéressent moins aux producteurs du défilé qu’à la réception de ce dernier dans le public, que ce soit les spectateurs "ordinaires» ou les élites. Ces chapitres analysent ainsi les deux premières grandes menaces au projet initial de la fête du Travail: le développement d'une culture de consommation, favorisée par l'implication progressive des commerçants dans les défilés, et celui d'une culture des loisirs privés au sein des classes populaires qui se désintéressent progressivement de cette manifestation publique. L'apparition de fêtes du Travail concurrentes constitue la troisième grande menace au spectacle des travailleurs qualifiés. Le cinquième chapitre retrace ainsi le développement de la fête socialiste du $1^{\text {er }}$ mai et de celle de Saint-Joseph des syndicats catholiques lors du dimanche qui précède le premier lundi de septembre. Par le biais de ce chapitre, les auteurs affinent ainsi leur analyse des tensions qui traversent la classe ouvrière pendant tout le $\mathrm{xx}^{\mathrm{e}}$ siècle. Quant à 
la fête du Travail du mois de septembre, le sixième chapitre montre qu'elle n'est plus, dans les dernières décennies $\mathrm{du} \mathrm{xx}^{\mathrm{e}}$ siècle, qu'une simple journée de congé qui marque la fin des vacances, un «festival du magasinage " qui sert de prétexte aux grands magasins pour écouler leurs marchandises d'été. Toutes ces tendances expliquent pourquoi on cesse, malgré de brefs sursauts de militantisme au tournant des années 1920 et au milieu des années 1940, de défiler lors de la fête du Travail. Seules les villes de Toronto et Hamilton ont réussi, quoique très difficilement, à conserver cette tradition de la célébration publique du premier lundi de septembre.

Heron et Penfold réussissent très bien à confronter le projet initial de la fête des travailleurs de métiers aux tensions apportées par les conflits de classes, de genres et de "races" à l'intérieur comme à l'extérieur de la classe ouvrière. De plus, le fil argumentaire du développement de la culture de consommation, et de son effet délétère sur l'espace public, permet d'ajouter une dimension analytique qui élargit vraiment les horizons de cette recherche. Il en ressort une étude très originale, passionnante, qui suscite chez le lecteur de nombreuses réflexions. Parmi celles-ci, j'aimerais m'attarder au concept de respectabilité, un concept polysémique qui désigne un ensemble de normes associées à l'homme-pourvoyeur blanc - ordre, sobriété, prévoyance, etc.- qui dicte les comportements autant dans la sphère publique que dans la sphère privée. Plutôt que de venir enrichir une réflexion déjà fort nuancée sur l'expérience ouvrière du défilé, il me semble que le recours continuel à ce concept en simplifie quelque peu la portée. Bien sûr, les auteurs soulignent que la définition de la «respectabilité» n'est pas fixée pour toujours et qu'elle est l'objet de débats à partir du xix ${ }^{\mathrm{e}}$ siècle. Toutefois, cette piste n'est pas suffisamment explorée et, au final, la respectabilité semble n'être le produit que du besoin de la partie organisée de la classe ouvrière de justifier son existence politique, économique, culturelle et sociale aux yeux des classes moyennes et bourgeoises.

Par exemple, les auteurs déplorent que les préoccupations entourant le port du chapeau lors des défilés ouvriers n’ont jamais dépassé les débats "étroits sur la mode» pour déboucher sur une remise en question de la respectabilité elle-même. Est-il possible que ces questions - sur le port des chapeaux, des insignes, des habits, etc. - qui nous apparaissent si triviales aujourd'hui aient eu une signification plus importante que ne le laisse croire la thèse de la respectabilité ? Ces préoccupations sont-elles le simple résultat de la «fausse» conscience ouvrière, modulée par les attentes de 
la société bourgeoise? Peut-on proposer d'y voir, dans un contexte où l'association s'impose difficilement au sein de la classe ouvrière, une des composantes d'une morale exigeante visant à rappeler continuellement la primauté des intérêts collectifs sur les intérêts particuliers des ouvriers? Porter un chapeau, porter un insigne, porter un habit, marcher en rang, ne pas être ivre, ne pas cracher par terre, bref les multiples règlements que les membres s'imposent eux-mêmes par le biais de leurs associations, témoignent à mon avis d'une utilisation populaire du discours de la respectabilité qui ne se résume pas à un désir de reconnaissance sociale dans l'espace public bourgeois. Ne s'agit-il pas aussi d'une tentative - même maladroite - de répondre aux nombreux problèmes que pose la construction de la solidarité dans une société de marché centrée sur la liberté individuelle? En ce sens, le défilé joue un rôle important non seulement pour la reconnaissance sociale, mais également comme un rituel populaire afin de rappeler le besoin de subordonner l'expression de l'individualité aux exigences solidaires du collectif, un enjeu crucial à une époque où la société de marché repose sur la concurrence de tous contre tous. Évidemment, il ne s'agit pas de remettre en cause le fait que le discours sur la respectabilité - de l'homme pourvoyeur blanc - ait également nourri un accès inégalitaire à l'espace public, notamment pour les femmes. Il s'agit plus simplement de souligner qu'une plus grande attention à ces multiples usages de la respectabilité aurait pu enrichir cette réflexion déjà fort stimulante sur les contradictions au cœur de l'espace public moderne.

Martin Petitclerc

Département d'histoire

Université du Québec à Montréal

PALMER,Alexandra, dir., Fashion:A Canadian Perspective (Toronto, University of Toronto Press, 2004), $382 \mathrm{p}$.

Très peu d'ouvrages s'intéressent à la mode canadienne. Le défi relevé par Alexandra Palmer est d'autant plus louable que le sujet est abordé sous plusieurs aspects. Que ce soit dans les contextes économique, social et culturel, ou sous l'angle de la haute couture et de la production de masse, ou de l'impact de sa diffusion dans les médias, la mode est étudiée en regard de la spécificité canadienne. Quinze auteurs ont apporté leur contribution à la rédaction de cet ouvrage divisé en quatre parties. Les essais, très documentés, dressent un large portrait du secteur de la 